\title{
Smart Container Security: the E-seal with RFID Technology
}

\author{
Jin Zhang \& Cuifen Zhang \\ Institute of Computer Science and Technology, Dalian Maritime University, Dalian 116026,China \\ E-mail: zbw0615@126.com
}

\begin{abstract}
In order to protect cargo from damage, theft, and terrorist threats, business and government turn to wireless sensors and RFID tags, and tradition container is replaced by smart container. In this paper, the basic technical features of RFID systems are described and linked to the practical applications. This paper will also determine how the technologies perform in the real-world operational environments and evaluate the various trade-offs that exist with E-seal design.
\end{abstract}

Keywords: Smart container, RFID technology, Electronic Container Seal (E-seal)

\section{Background}

Cargo seals are more common in international trade than for domestic shipments. This reflects the historical and continuing importance of Customs duties and cross-border smuggling. Manual cargo seals have long been part of good security practice. Their principal purpose is to assure carriers, beneficial owners of cargo.

Manual seals vary widely in the degree of protection they offer. Many factors affect protection, including the design, materials, and construction of the locking device, and the design and materials in the hasp, bolt, or cable. The trade abounds with tales of popular manual seal designs that have been copied with cheap materials. There are no international standards for manual seals.

Good seal practices improve the odds but cannot guarantee shipment integrity. Clever miscreants can defeat seals in numerous ways, such as cutting holes in the side or top of a container and then repairing it. However, the effectiveness of seal programs seems more affected by poor practices than by unusually skillful criminals.

\section{Smart container}

Cargo container with smart systems alters globe network in real time about security beaches. The smart system is open, flexible and modular in design. This will enable users to configure other sensors or automatic identification technologies in combinations applicable to their security or cargo management needs. Other container sensors can include hazardous chemical detection and various physical parameters including temperature, light, vibration, shock, atmospheric pressure and so on.

\section{Electronic Container seal (E-seal)}

E-seal ensure that ever-increasing cargo loads also have far greater protection. These seals, combining robust mechanical parts with sophisticated sensors, deliver a highly cost-effective solution for the cargo industry. An E-Seal is a radio frequency device that transmits container information as it passes a reader device, and issues alerts and error conditions if the container has been tampered with or damaged. E-Seals serve both commercial and security interests by tracking commercial container shipments from their point of origin, while en route, and to their final destination and point of customs clearance.

\subsection{E-seal technology}

E-seals marry manual seal elements with electronic components to measure seal integrity, store data, and provide communications. Some designs use infrared signals and others use direct contact communications technologies, but radio frequency identification (RFID) is the most common choice. Most e-seal designs automate the essential functions of seal checking and reporting in order to remove human intervention. ${ }^{\text {[a] }}$

RFID has long been touted as the future of logistics for all companies by allowing retailers and suppliers to track goods throughout the supply chain. Global logistics company Schenker is testing the use of radio frequency identification (RFID) technology to track containers used for overseas shipments.

\section{RFID Seal}


RFID is the system technology that recognizes a human or material by radio information communication and writes additional information through the media of a card/tag that is built-in IC chip or resonance material.

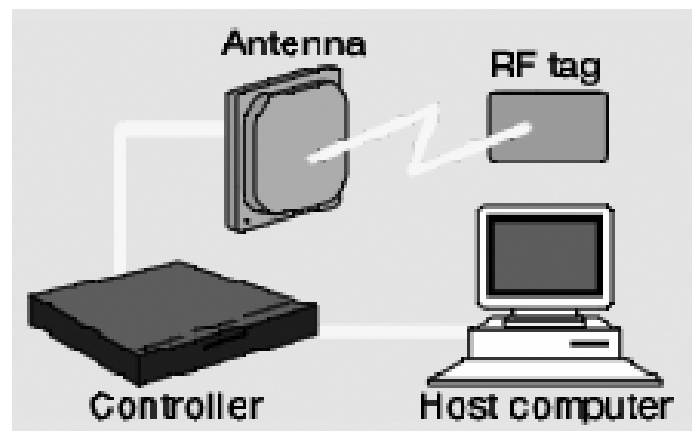

Figure 1.

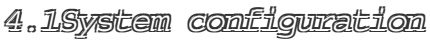

The purpose of the system is to communicate data between informational medium (that is called "RF tag", "Transponder" of the shape of the card/tag) and an interrogator (that is called "Reader", "Interrogator") by the electric wave.

\subsection{Features and Classification}

Recognizable from a distance; Recognizable through an obstacle; Reading two or more tags simultaneously is possible; Writing additional information is also possible; RF tag is reusable.

There are two main types of RFID tags and seals, passive and active.

Passive seals do not initiate transmissions - they respond when activated by the energy in the signal from a reader. Interrogated by a reader, a passive seal can identify itself by reporting its "license plate" number, analogous to a standard bar code. The tag can also perform processes, such as testing the integrity of a seal. The beauty of a battery-free passive seal is that it can be a simple, inexpensive, and disposable device. Passive RFID seals can carry batteries for either or both of two purposes. The first is to aid communication by boosting the strength of the reflective signal back to the reader. This capability need not add much cost. The second purpose is to provide power so functions can be performed out of the range of readers.

Active seals can initiate transmissions as well as respond to interrogation. All active tags and seals require on-board power, which generally means a battery.

All active RFID electronic seals on or approaching the market monitor seal integrity on a near-continuous basis, and most capture the time of tampering and write it to an on-board log. Some can accept GPS and sensor inputs, and some can provide live "mayday" tampering reports as the events happen, mostly within specially equipped terminals.

Passive vs. Active RFID seals. One may look at the trade-offs between these technologies from theoretical and practical perspectives. Theoretically, the only difference between passive and active tags and seals is the ability to initiate communications from the tag-distinction that means passive RFID tags could not initiate mayday calls. However, a designer could add on-board power to a passive tag, match other functionality and, setting aside regulatory, safety, and cost issues, increase read range and directional flexibility by increasing power and adding antennas.

\subsection{Standards and Frequencies}

Adoption of RFID in supply chain and security applications is hampered by a lack of standards and by what some call "the frequency wars." The two issues are interrelated. Standards for electronic seals address technical protocols, interfaces, and frequencies. There are three related items.

ISO 10374 is the existing voluntary standard for RFID automatic identification of freight containers. It is a dual frequency passive read-only standard that includes $850-950$ and 2400-2500 MHz. Globally, only two carriers use these tags, one primarily on chassis and the other on chassis, ocean containers, and many dray trucks.

ISO 18185 is a Draft International Standard for electronic container seals. It includes passive and active protocols, enabling both simple low cost and more robust seals. The active protocols have been the focal point for "the frequency wars" in terms of freight containers.

ISO 23359 is a New Work Item for read/write RFID for freight containers. Work started on this project in June 2002, and it seems likely to build closely on the draft seal standard.

Frequency choice begins with technical performance but includes political and regulatory issues. In crowded freightoriented environments such as warehouses and terminals, the most effective frequencies appear to be between 100 and 1000 
MHz. Frequencies below $100 \mathrm{MHz}$ lose range rapidly because of inductive coupling or noise from electrical coupling. Frequencies above $1000 \mathrm{MHz}$, with shorter wavelengths, cannot wrap or diffract around objects such as vehicles and freight containers-they become more line-of-sight and subject to blind spots.

\section{Looking Ahead}

Container shipping is a critical component of global trade: about $90 \%$ of global trade is transported in cargo containers. Globalization and market deregulation have also led to advances in security devices and instruments and their strategic integration within the logistics sector. The market for E-seal that may improve both freight transportation security and productivity is in its early stages. We will become a big winner of market in development through relatively early stages of use.

\section{References}

Hu, Jianyun, He, Yan \& Min, Hao. (2006). Design and Analysis of Analog Front-End of Passive RFID Transponders. Chinese Journal of Semiconductors. Vol.27 No.6 p.999-1005

Jung-Hyun Cho, et. (2005). An analog front-end IP for 13.56MHz RFID interrogators. Proceedings of the 2005 conference on Asia South Pacific design automation. p. 1208 - 1211.

Le-Pong Chin \& Chia-Lin Wu. (2004). The Role of Electronic Container Seal (E-Seal) with RFID Technology in the Container Security Initiatives. MEMS, NANO and Smart Systems, 2004. ICMENS 2004. Proceedings. 2004 International Conference on Aug. p.116- 120.

(2003). International standard ISO/IEC 14443 -1, -2, -3, International Standardization Organization. April. 